\title{
Sensitivity for Multivariate Calibration based on Multilayer Perceptron Artificial Neural Networks
}

Fabricio A. Chiappini ${ }^{1,4}$, Franco Allegrini ${ }^{2}$, Héctor C. Goicoechea ${ }^{1,4, *}$ and Alejandro C. Olivieri ${ }^{3,4, *}$

${ }^{1}$ Laboratorio de Desarrollo Analítico y Quimiometría (LADAQ), Cátedra de Química Analítica I, Facultad de Bioquímica y Ciencias Biológicas, Universidad Nacional del Litoral, Ciudad Universitaria, Santa Fe (S3000ZAA), Argentina.

${ }^{2}$ Laboratorios Fox, Moreno 678, Venado Tuerto, Argentina.

${ }^{3}$ Departamento de Química Analítica, Facultad de Ciencias Bioquímicas y Farmacéuticas, Universidad Nacional de Rosario, Instituto de Química de Rosario (IQUIR-CONICET), Suipacha 531, Rosario (S2002LRK), Argentina.

${ }^{4}$ Consejo Nacional de Investigaciones Científicas y Técnicas (CONICET), Godoy Cruz 2290 CABA (C1425FQB), Argentina.

*E-mails: olivieri@iquir-conicet.gov.ar (A.C. Olivieri), hgoico@fbcb.unl.edu.at (H.C. Goicoechea) 
This document contains supporting information on the following subjects

1. Simulated data. Validation of the proposed equations through Monte Carlo simulations.

2. Experimental data. Validation of the proposed equations through a bootstrap methodology. 


\section{Simulated data. Validation of the proposed equations through Monte Carlo simulations.}

One way to check that the derived equations for the calculation of the sensitivity parameter are correct [Eq. (6) in the main text] is by means of noise addition Monte Carlo calculations. This involves adding a small amount of noise to test sample spectra, predicting the analyte concentrations and registering the statistical distribution of the latter values.

In the context of analytical calibration, the general definition of sensitivity (SEN) can be conceived as:

$$
\mathrm{SEN}=\frac{\sigma_{x}}{\sigma_{y}}
$$

where $\sigma_{x}$ represents the input noise (uncertainty in signal) and $\sigma_{y}$ the uncertainty in predicted concentration. It is important to remark that, in the case of a non-linear calibration, the SEN is sample-dependant, i.e., the slope of the function establishing the relation between signal and concentration is not constant.

Under the hypothesis of a noiseless calibration, it is possible to explore the propagation of uncertainty from the signal to the predicted concentration by adding a small amount of independently and identically distributed (iid) noise to the spectrum of a generic test sample. In this sense, Monte Carlo simulation yields an empirical estimation of the sensitivity of a sample when its concentration is predicted by the multivariate model. The Monte Carlo estimations of SEN can be then compared with the theoretical calculations.

In the present work, four different simulated non-linear systems were generated, i.e. quadratic, irrational, sigmoidal and logarithmic. In each case, a proper MLP calibration model was obtained starting from a noiseless calibration dataset (4 components in the concentration range of 1-5 arbitrary units, 625 samples from a full factorial design). Additionally, test datasets of 500 replicates were generated. For each system, 55 different datasets were simulated by varying the concentration of the analyte in 11 equidistant points within the concentration range and five different percentage of iid noise (27,000 test samples were processed in total). As an example, the results of Monte Carlo calculations for the quadratic system are shown below. Spectral data are depicted in Figure S1: S1A, unit-concentration spectra of the four components used to 
generate the simulated systems (the black spectrum corresponds to the analyte of interest whereas the remaining spectra represent the interferents), S1B, selected examples of 30 calibration spectra, and S1C, the spectrum of a generic test sample with $0.1 \%$ of the signal maximum in calibration set.
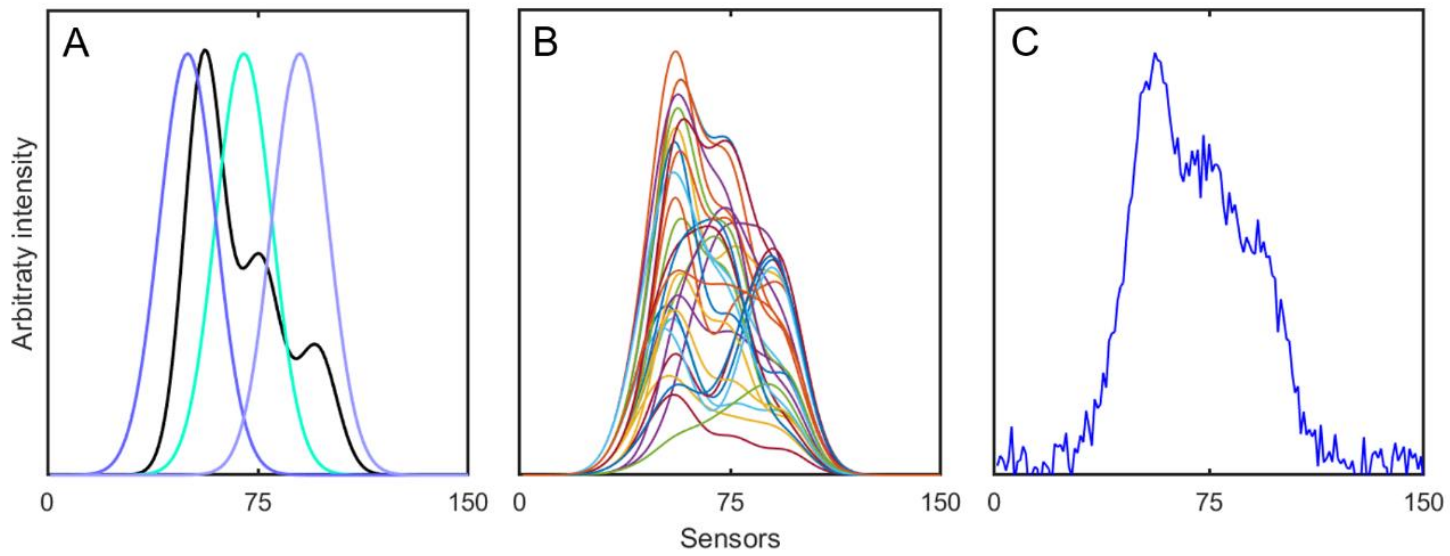

Figure S1. Simulated spectral data. A. Unit-concentration spectra of the four components used to generate the simulated systems. B. Selected examples of 30 calibration spectra. C. The spectrum of a generic test sample with $0.1 \%$ of the signal maximum in calibration set as an amount iid noise.

Calibration data $\left(\mathbf{X}_{\text {cal }}\right)$ was used to train an MLP model, whose optimum architecture was of 4-2-1 for the input, hidden and output layers, respectively. Each test dataset was used to make predictions of the analyte. In each prediction cycle, 500 test samples of a given concentration level of the analyte were predicted (500 replicates). A value of $\sigma_{y}$ was computed from the standard deviation of the output concentrations at different levels of iid noise, i.e. $0.01,0.05,0.1,0.5$ and $1 \%$ of the signal maximum in calibration set $\left(\max \left(\mathbf{X}_{c a l}\right)\right)$. These levels of noise constituted the values of $\sigma_{x}$. Hence, according to the general definition of SEN from Eq. (S1), a relationship between $\sigma_{y}$ and $\sigma_{x}$ was established as:

$$
\sigma_{x}=\operatorname{SEN} \sigma_{y}
$$

At each concentration level, SEN was obtained from the estimated slope of a linear regression of $\sigma_{x}$ vs $\sigma_{y}$. The obtained results for the quadratic system are graphically summarized in Figure S2. 

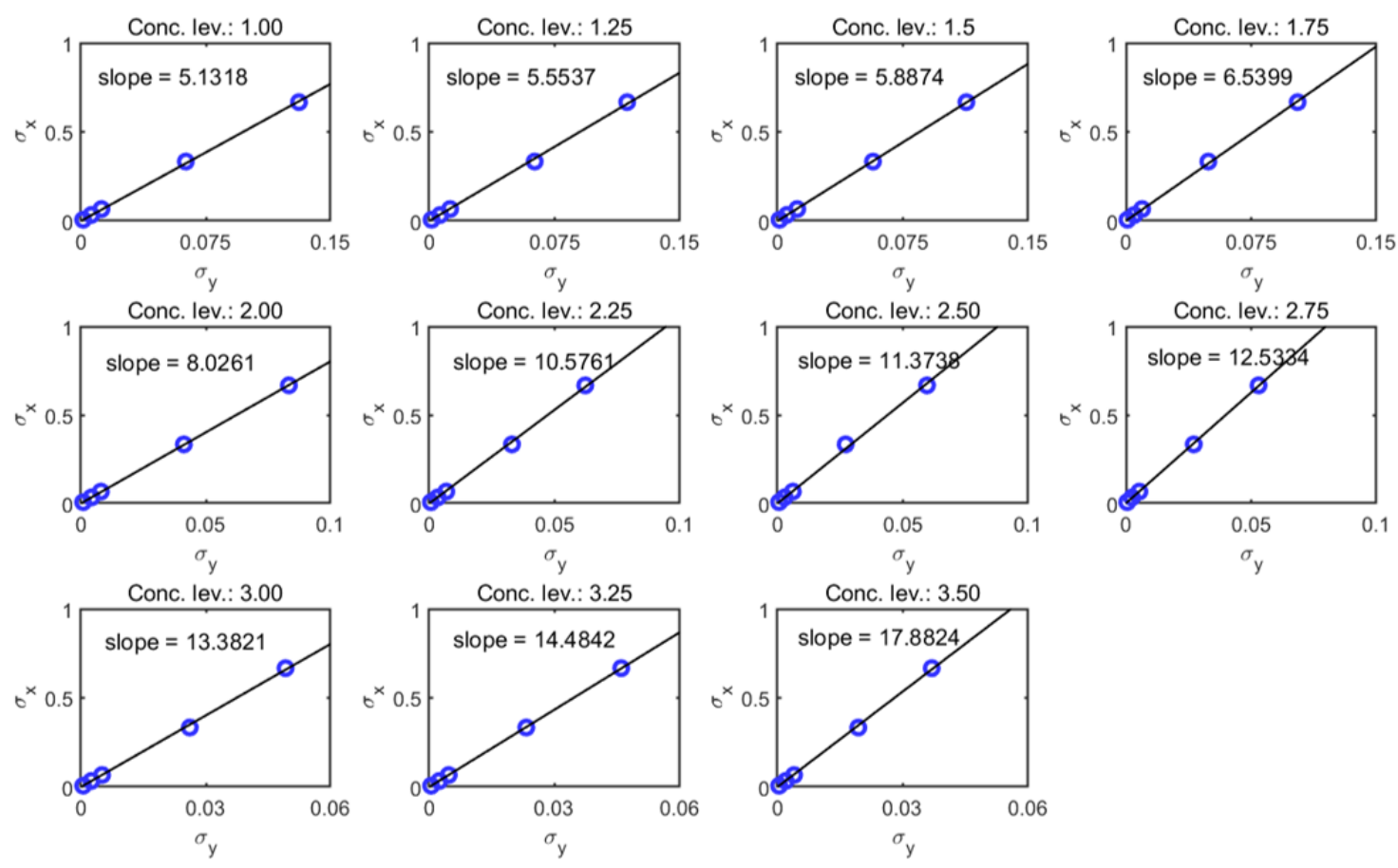

Figure S2. Graphical outputs for the calculation of SEN parameters via Monte Carlo simulation. Each subplot represents the regression line obtained after plotting the values of $\sigma_{x}$ (iid added noise to test samples) vs $\sigma_{y}$ (standard deviation of 500 prediction replicates) for each concentration level. 


\section{Experimental data. Validation of the proposed equations through a bootstrap methodology.}

In the case of the experimental dataset, the proof of concept for the validation of the proposed equations for the estimation of SEN was carried out by implementing a bootstrap strategy. The bootstrap is a data resampling methodology that enables one the estimation of statistical parameters from an unknown distribution. This approach was implemented to obtain an approximation of the uncertainty in prediction associated to each sample in the experimental dataset, i.e., the standard deviation of the generated distribution obtained after 1000 bootstrap prediction cycles $\left(\sigma_{y}^{b o o t}\right)$. In addition, the value of uncertainty in signal, i.e., the instrumental noise, was estimated from the error obtained after PCA decomposition of the spectral data matrix with the optimum number of components $\left(\sigma_{x}^{\mathrm{PCA}}\right)$. Thus, in agreement with the general definition of SEN [Eq. (S1)], a value of bootstrap sensitivity $\left(\mathrm{SEN}_{\text {boot }}\right)$ for each sample was obtained as the ratio of $\sigma_{x}^{\mathrm{PCA}}$ and $\sigma_{y}^{b o o t}$.

Briefly, the implemented methodology consisted in separating $25 \%$ of the samples for prediction (validation sub-set). The remaining $75 \%$ (calibration sub-set) was refilled by randomly re-sampling a proper number of samples of the same set to reach $100 \%$ of the original number of samples. This procedure was iteratively repeated (1000 times) by training a MLP with a fixed architecture (previously optimized) and using the validation set to make predictions. The whole process was repeated for four different validation sets (left-out samples), in order to estimate $\sigma_{y}^{\text {boot }}$ of all samples in the dataset. As an example, Figure S3 shows the obtained histograms for 1000 bootstrap predictions of nine samples. 

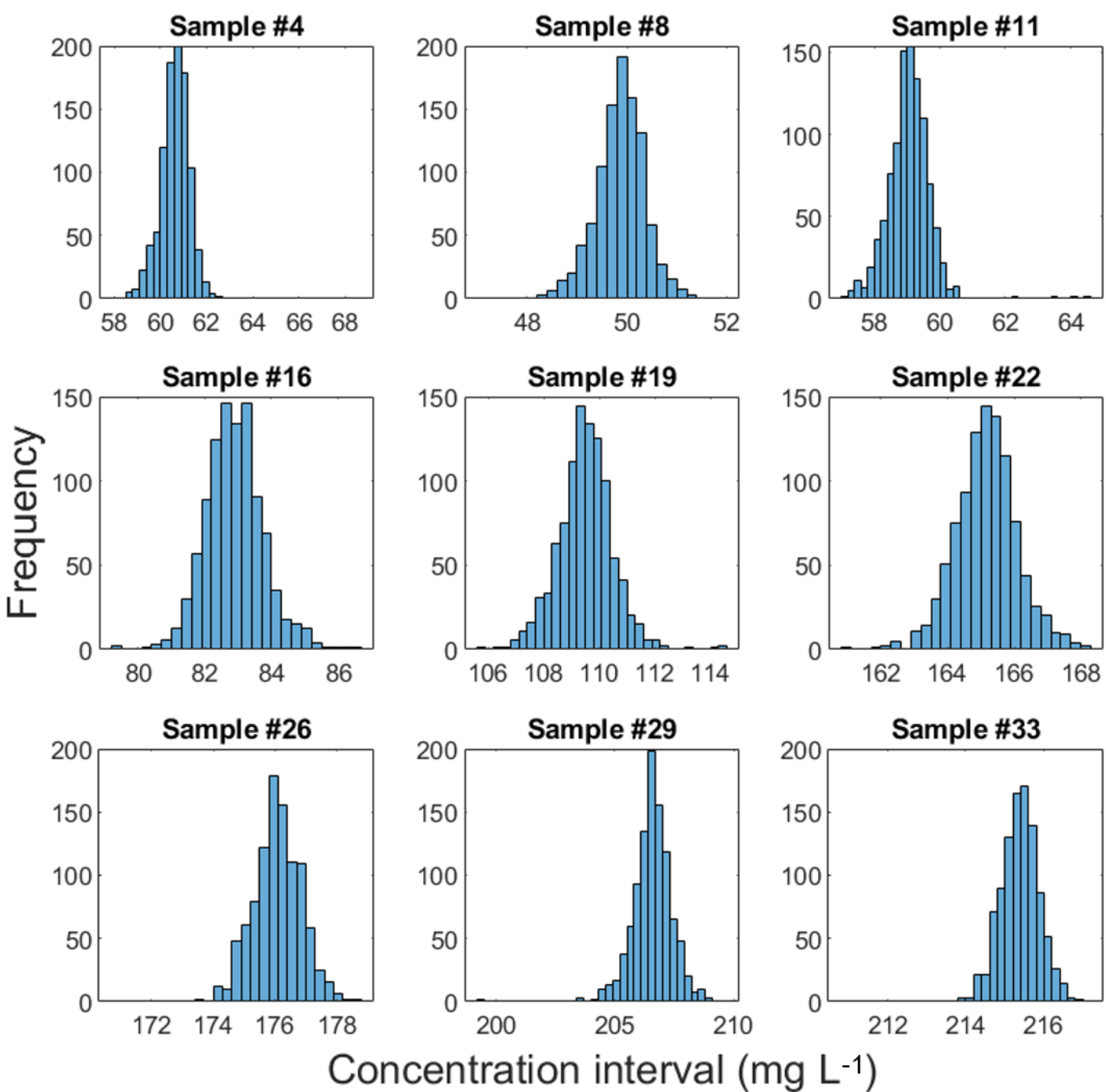

Figure S3. Histograms for 1000 bootstrap predictions of nine samples from experimental dataset (fluorescence) for the estimation of $\sigma_{y}^{b o o t}$ for empirical calculation of SEN parameter. 